\title{
The role of hydrophobic hydration in the free energy of chemical reactions at the gold/water interface: size and position effects
}

\author{
Alessandra Serva, ${ }^{1,}$ a) Martina Havenith, ${ }^{2,3}$ and Simone Pezzotti ${ }^{2,}$ b) \\ ${ }^{1)}$ Sorbonne Université, CNRS, Physico-chimie des Electrolytes et Nanosystèmes Interfaciaux, PHENIX, \\ F-75005 Paris, France \\ ${ }^{2)}$ Department of Physical Chemistry II, Ruhr University Bochum, 44780 Bochum, \\ Germany \\ ${ }^{3)}$ Department of Physics, Technische Universität Dortmund, 44227 Dortmund, \\ Germany
}

Metal/water interfaces catalyze a large variety of chemical reactions, which often involve small hydrophobic molecules. In the present theoretical study we show that hydrophobic hydration at the $\mathrm{Au}(100) /$ water interface actively contributes to the reaction free energy by up to several hundreds of meV. This occurs either in adsorption/desorption reaction steps, where the vertical distance from the surface changes in going from reactants to products, or in addition and elimination reaction steps, where two small reactants merge into a larger product and viceversa. We find that size and position effects cannot be captured by treating them as independent variables. Instead, their simultaneous evaluation allows to map the important contributions, and we provide examples of their combinations for which interfacial reactions can be either favoured or disfavoured. By taking a $\mathrm{N}_{2}$ and a $\mathrm{CO}_{2}$ reduction pathway as test cases, we show that explicitly considering hydrophobic effects is important for the selectivity and rate of these relevant interfacial processes.

\section{INTRODUCTION}

Metal/water interfaces provide a unique environment for a large variety of chemical reactions, with applications in e.g. electrochemistry and heterogeneous catalysis. $^{1-6}$ The ability of these interfaces to modulate reaction free energies, and therefore selectivity and rate, is usually evaluated in terms of the interactions between the reactive species and the surface. ${ }^{7-10}$ However, interfacial water also contributes to the reaction free energies by hydrating reactants, intermediates and products. ${ }^{11-15}$ In interfacial reactions involving small hydrophobic molecules, such as $\mathrm{CO}_{2}$ and $\mathrm{N}_{2}$ reduction, understanding how hydrophobic hydration free energies at the interface modulate thermodynamics could hence provide novel opportunities for improving reactions efficiency and selectivity.

From a theoretical point of view, while the catalytic role of the solid surface is generally evaluated with accuracy by high level Density Functional Theory (DFT) calculations, ${ }^{7,16-20}$ the free energy contributions arising from changes in hydrophobic hydration during chemical reactions are often overlooked. One reason is that, due to the high computational cost of DFT approaches, interfacial water is often either represented in the calculations by implicit models or only few molecules are explicitly treated. However, hydrophobic hydration free energy depends on the characteristic of the water H-Bond network at the interface. ${ }^{21,22}$ Indeed, the hydration of a hydrophobic molecule is determined by the process of perturbing the water $\mathrm{H}$-Bond network

\footnotetext{
a) Electronic mail: alessandra.serva@sorbonne-universite.fr

b) Electronic mail: simone.pezzotti@rub.de
}

and creating a cavity in the liquid. ${ }^{23,24}$ Recent advances in molecular dynamics (MD) simulations that include multiple layers of explicit water allow to more accurately describe the hydration contributions to interfacial chemical reactions. ${ }^{25-30}$ However, these simulations still face challenges related to the length (nanometers) and time scales (up to tens of nanoseconds) needed to describe the changes in the water network in contact with metal surfaces. $^{21}$

In a recent work, based on constant potential classical MD simulations we have shown that the mechanisms and energetics for hydrophobic hydration at an electrified $\mathrm{Au}(100)$ /water interface differ from what is known in the bulk. ${ }^{22}$ As a consequence, we have proposed an adaptation of the Lum-Chandler-Weeks theory that allows quantifying the free energy cost of cavity formation (i.e. the free energy cost to hydrate ideal hard-sphere solutes) at the interface directly from the well-known free energy cost in the bulk plus an interface-dependent correction term obtained from the simulations. ${ }^{22}$ While hydrophobic hydration in the bulk only depends on the size of the cavity formed by the hydrophobe, the correction term also depends on the distance of such cavity from the metal surface. As a result, both the size of the molecule and its distance from the surface largely modulate hydrophobic hydration free energies. By applying the adapted theory to a simple model chemical reaction, we have shown that the outer-sphere (i.e separated from the surface by one water layer) addition between two ideal hydrophobes of size comparable to a $\mathrm{CO}$ molecule suffer from an energetic penalty imposed by the high cost of large cavities formation at the $\mathrm{Au}(100) /$ water interface. ${ }^{22}$ However, since hydration free energies can largely vary within few angstroms at the interface, rationalizing hydrophobic effects requires going beyond the simplification that 
reactants and products are ultimately confined in the outer layer. To this end, we here quantitatively evaluate how the hydrophobic hydration contribution to model chemical reactions changes as a function of both the size of the reactive species and their vertical distance from the surface. We also provide two examples of chemical reactions where the unravelled hydrophobic effects play an important role.

\section{METHODS}

We consider in the following model reactions occurring at the $\mathrm{Au}(100) /$ water interface where reactants (R) and products $(\mathrm{P})$ are ideal spherical hydrophobes. The system is considered at its point of zero charge (PZC), which corresponds to an applied voltage of $0 \mathrm{~V}$ in our model. Since we are dealing with ideal hydrophobes, the free energy of the reaction is solely determined by the difference in the free energy cost to form the cavity of the products and of the reactants:

$$
\begin{aligned}
\Delta F_{\text {reax }}= & \Delta F_{\text {cavity }}= \\
& =\sum_{i=1}^{N_{P}} \Delta \mu_{v}^{i n t}\left(r_{i}, z_{i}\right)-\sum_{j=1}^{N_{R}} \Delta \mu_{v}^{i n t}\left(r_{j}, z_{j}\right)
\end{aligned}
$$

where $\mathrm{N}_{R}$ and $\mathrm{N}_{P}$ are the number of reactants and products respectively, while $\Delta \mu_{v}^{i n t}$ is the free energy cost of cavity formation at the interface. The $\Delta \mu_{v}^{\text {int }}$ value (at PZC) for any reactant or product solely depends on its radius $(r)$ and vertical distance from the surface $(z)$ and is obtained from the adapted LCW theory we have introduced in Ref. 22. According to eq.1, $\Delta \mathrm{F}_{\text {cavity }}$ is function of the size and position of all reactive species, and in the following we restrict our attention to values of $\mathrm{r}<3.0 \AA$ and $-1.0 \AA<\mathrm{z}-\mathrm{z}^{*}<1.0 \AA$, where $\mathrm{z}^{*}$ identifies the most stable position for ideal hydrophobes at the $\mathrm{Au}(100)$ /water interface. As shown in Ref. 22, $\mathrm{z}^{*}$ is a function of the cavity radius, $r$, and it is equal to the z-position of the water adlayer in direct contact with Au plus r. Therefore, as illustrated in the snapshot of Figure $1, \mathrm{z}^{-} \mathrm{z}^{*}=0 \AA$ corresponds to an outer-layer adsorption, where the hydrophobe is separated from the $\mathrm{Au}$ surface by one water layer (the adlayer), while $\mathrm{z}-\mathrm{z}^{*}=$ -1.0 A corresponds to an inner-sphere adsorption, the hydrophobe being in direct contact with the surface.

In order to estimate how much hydrophobic hydration free energy contributes to the "real" chemical reactions discussed in section II C, only the knowledge on the position and size of the reactive molecules is required; from this, $\Delta \mathrm{F}_{\text {cavity }}$ is obtained via eq. 1 . The position of the cavities formed by reactants and products are known from Refs. 17,31 (vide infra), while their sizes are approximated with spheres. The radius of the spherical cavity formed by a molecule can be considered as the average distance between the molecule center of mass

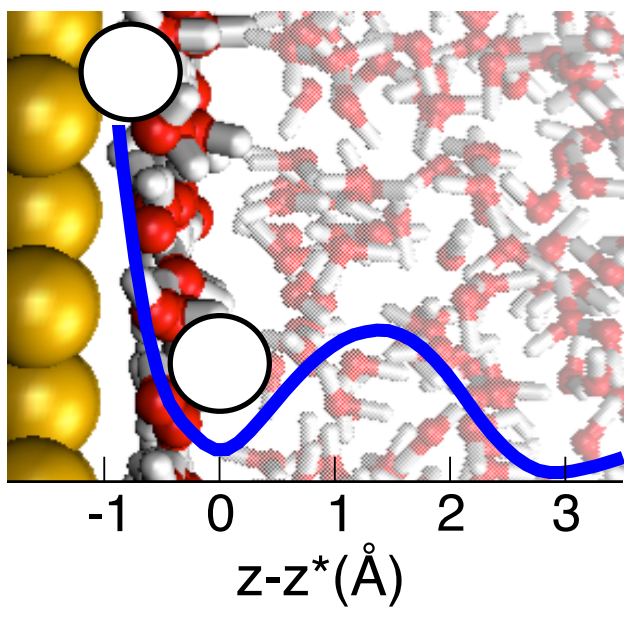

FIG. 1. MD snapshot of the interface between Au(100) (yellow) and water (red, oxygens and white, hydrogens) at PZC. Water molecules belonging to the adlayer (in direct contact with $\mathrm{Au}$ ) are highlighted. The blue curve represents the free energy profile to bring an ideal hydrophobe from the bulk to the surface (see Ref. 22 for more details), while the white circles at $\mathrm{z}-\mathrm{z}^{*}=0$ and $-1.0 \AA$ illustrate the outer-layer and inner-layer adsorption for an ideal hydrophobe, respectively.

and the oxygen centers of the closest water molecules; the distance is deduced from the radial distribution functions, RDFs. ${ }^{32,33}$ For molecules that deviate from spherical shape, the radius of the equivalent sphere is considered. This approximation has been validated in the case of the $\mathrm{CO}$ molecule by quantitatively comparing its adsorption profile at the $\mathrm{Au}(100) /$ water interface to the one of a cavity of $2.5 \AA$ radius (see ref. 22). The RDFs values are taken from refs. $32,34,35$, and the cavity radii obtained are $2.5 \AA$ for $\mathrm{CO}$ and $\mathrm{N}_{2}, 3.1 \AA$ for $\mathrm{C}_{2} \mathrm{H}_{4}$ and $3.0 \AA$ for $\mathrm{N}_{2} \mathrm{H}_{4}$. Molecules that differ only for one $\mathrm{H}$ atom are assumed to form cavities of the same radius, while the $\mathrm{OCCOH}^{*}$ intermediate (which is also considered in the following) is assumed to have the same volume as two CO units. It is important to stress here that such values are approximations, and for a more accurate quantification of the hydrophobic contribution the exact cavity volume, including its deviation from spherical symmetry, must be considered.

\section{RESULTS AND DISCUSSIONS}

Chemical reactions where hydrophobic effects are expected to play an important role are those where either (i) the vertical distance from the surface or (ii) the size of the product(s) is different from the one of the reactant(s). Condition (i) is satisfied in adsorption/desorption reaction steps, where the reactive species move across the interface; condition (ii) in addition and elimination reaction steps, where two reactive species merge into a larger product and viceversa. 


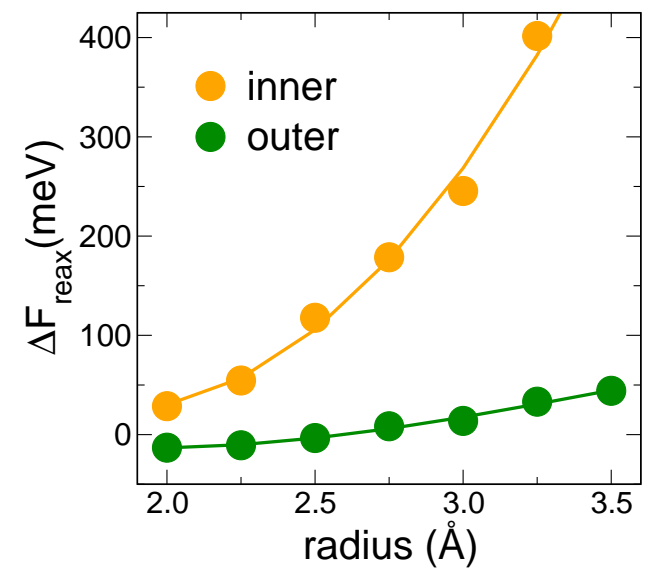

FIG. 2. $\Delta \mathrm{F}_{\text {reax }}$ for the adsorption (from bulk to interface) of a spherical ideal hydrophobe, as a function of its radius. Two distinct adsorption processes are compared, bringing the hydrophobe either in the inner-layer $\left(\mathrm{z}-\mathrm{z}^{*}=-1.0 \AA\right.$, orange) or in the outer-layer $\left(\mathrm{z}-\mathrm{z}^{*}=0.0 \AA\right.$, green).

\section{A. Adsorption/desorption reaction steps}

We start by considering the adsorption/desorption of hydrophobic species to/from the surface. Both the size of the hydrophobe and whether it is adsorbed in the inner or outer layer can influence the thermodynamics. In order to quantify these effects, we consider a model adsorption process, where an ideal hydrophobe $(\mathrm{R})$ is moved from the bulk to the interface:

$$
R^{b u l k} \rightarrow R^{i n t}
$$

with associated reaction free energy:

$$
\Delta F_{\text {reax }}=\Delta F_{\text {cavity }}=\Delta \mu_{v}^{i n t}\left(r_{R}, z\right)-\Delta \mu_{v}^{b u l k}\left(r_{R}\right)
$$

where $\mathrm{r}_{R}$ is the size of the ideal hydrophobe, and $\mathrm{z}$ $\mathrm{z}^{*}=-1.0$ and $0 \AA$ for the adsorption in the inner- and outer-layer, respectively (see illustration in Fig. 1). The $\Delta \mathrm{F}_{\text {reax }}$ values in Figure 2 show that the adsorption in the outer-layer (green) always requires lower free energies than in the inner-layer (orange), and the difference between the two becomes larger with increasing radii. For real hydrophobic molecules, this implies that the adsorption in the inner-layer can occur only if the molecule is sufficiently stabilized by the direct interaction with the surface (chemi- or physisorption), while a free energy cost is paid in term of hydrophobic hydration. Moreover, our results suggest that the adsorption in the inner-layer can efficiently activate the reactants involved in any interfacial chemical process by bringing them to a high energy (unfavourable) solvation state and making them more reactive. If no additional stabilization is involved, the desorption of a spherical product from the surface (which free energy is minus the $\Delta \mathrm{F}_{\text {reax }}$ values reported in Figure 2) is favoured by hydrophobic hydration.

\section{B. Addition/elimination reaction steps}

When two ideal spherical hydrophobes (reactants, $\mathrm{R}_{1}$ and $\mathrm{R}_{2}$ ) addition to form a larger cavity (product, $\mathrm{P}$ ), with its volume being the sum of the volumes of $R_{1}$ and $\mathrm{R}_{2}$

$$
R_{1}+R_{2} \rightarrow P
$$

$\Delta \mathrm{F}_{\text {reax }}$ is expressed by:

$$
\begin{aligned}
\Delta F_{\text {reax }}=\Delta & F_{\text {cavity }}=\Delta \mu_{v}^{i n t}\left(r_{P}, z_{P}\right)+ \\
& -\Delta \mu_{v}^{i n t}\left(r_{R 1}, z_{R 1}\right)-\Delta \mu_{v}^{i n t}\left(r_{R 2}, z_{R 2}\right)
\end{aligned}
$$

where $r_{P}=\left(r_{R 1}^{3}+r_{R 2}^{3}\right)^{1 / 3}$. The full landscape of $\Delta \mathrm{F}_{\text {reax }}$ over its 5-dimensional domain $\left(\mathrm{r}_{R 1}, \mathrm{r}_{R 2}, \mathrm{z}_{R 1}, \mathrm{z}_{R 2}\right.$ and $\left.\mathrm{z}_{P}\right)$ has been mapped by combining eq. 5 and the adapted LCW theory (see Methods). We find a variation of $\Delta \mathrm{F}_{\text {reax }}$ of several hundreds of $\mathrm{meV}$, from a minimum of $-420 \mathrm{meV}$ to a maximum of $620 \mathrm{meV}$, indicating that hydrophobic hydration can provide large contributions to interfacial reactions. In the following we focus on relevant subdomains of the $\Delta \mathrm{F}_{\text {reax }}$ function, obtained by simultaneously exploring two dimensions while fixing the other three. This is required to map the important contributions, as considering one dimension only does not yield any relevant information (see Supplementary Figure S1).

\section{Effect of the vertical distance from the surface}

Firstly, $\Delta \mathrm{F}_{\text {reax }}$ is evaluated by fixing the radii of $\mathrm{R}_{1}$ and $\mathrm{R}_{2}$ to $2.5 \AA$ (approximately the size of a $\mathrm{CO}$ molecule ${ }^{22}$ ) and by varying the vertical distance of reactants and product from the surface.

The 2D-map of Figure $3 \mathrm{~A}$ is obtained by simultaneously moving the reactants $\left(\mathrm{z}_{R 1}=\mathrm{z}_{R 2}=\mathrm{z}_{R}\right)$ on the $\mathrm{x}$-axis, while the position of the product is varied on the y-axis. At a first glance, one can notice that any vertical distance from the surface has a huge impact on the free energy, which goes from $-200 \mathrm{meV}$ (dark blue) to $+450 \mathrm{meV}$ (dark red), even for very small displacements within a $1 \AA$ range. This means that addition reactions involving small hydrophobic molecules as $\mathrm{CO}$ or $\mathrm{N}_{2}$ can be either favoured or disfavoured by the hydrophobic term depending on subtle differences in the way reactants and products are adsorbed at the $\mathrm{Au}$ surface. In particular, the addition reaction is favoured when $R_{1}$ and $R_{2}$ are adsorbed to the surface $\left(z_{R}-z^{*}<\right.$ $-0.5 \AA)$, while the product that is formed desorbs from it $\left(\mathrm{z}_{P}-\mathrm{z}^{*}>-0.5 \AA\right)$, as illustrated by scenario I in Figure $3 \mathrm{C}$. The reason is that the solvation of the product cavity is stabilized in the outer-layer, while a much higher free energy cost is required to solvate the two reactants cavities in the inner-layer. Accordingly, if we now move from I to II in the map, we can see that when also the reactants are solvated in the outer layer, their 

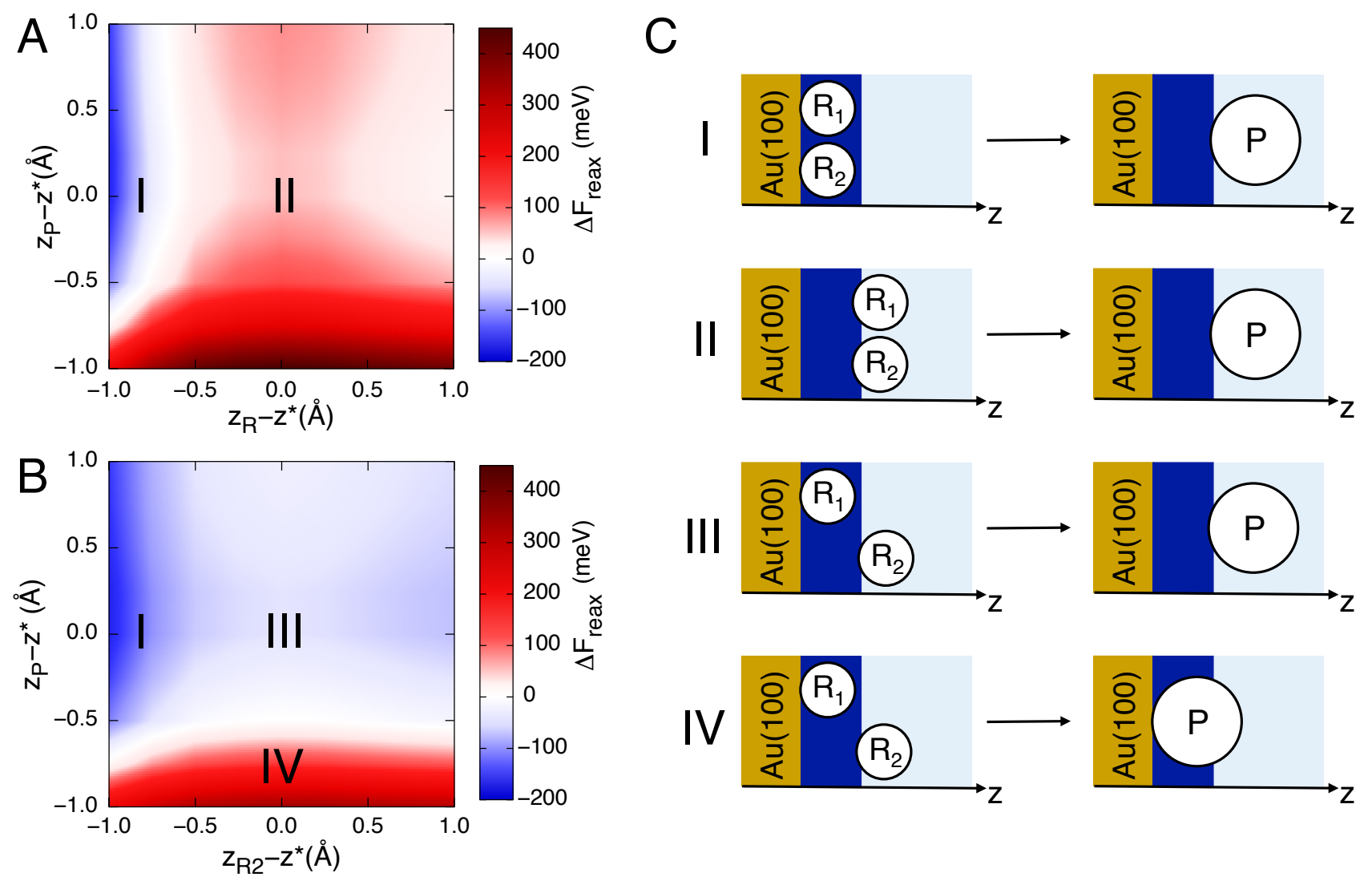

FIG. 3. A) $\Delta \mathrm{F}_{\text {reax }}$ (from dark blue to dark red) as a function of the position of the reactants, $\mathrm{z}_{R^{-}} \mathrm{z}^{*}$, which are moved

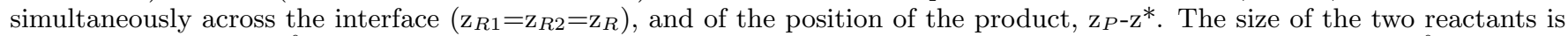
fixed to $\mathrm{r}_{R 1}=\mathrm{r}_{R 2}=2.5 \AA$. B) Same as A, but the position of one reactant is fixed in the inner-layer $\left(\mathrm{z}_{R 1}-\mathrm{z}^{*}=-1.0 \AA\right)$. Some of the relevant scenarios are marked with roman numbers (I-IV) and illustrated by the schemes in panel C.

addition is not favoured anymore and $\Delta \mathrm{F}_{\text {reax }}$ becomes positive. Finally, the addition reaction becomes even more disfavoured when $\mathrm{P}$ is adsorbed to the surface, whatever the position of the reactants (red stripe at $\left.\mathrm{z}_{P}-\mathrm{Z}^{*}<-0.5 \AA\right)$.

To complete our analysis on the effect of the position, in the 2D-map of Figure 3B we now fix $z_{R 1}-z^{*}=$ $-1.0 \AA$ (i.e one reactant is in the inner layer), and we let $\mathrm{R}_{2}$ and $\mathrm{P}$ free to move. Again we can see that the reaction is most favourable when the product is solvated in the outer-layer, with the vertical distance of the reactants from the surface also playing a crucial role. This can be quantified by comparing scenarios I, II and III in the two maps, which only differ for the position of the reactants. Scenario I, with both reactants in the inner-layer, provides $\Delta \mathrm{F}_{\text {reax }} \simeq-200 \mathrm{meV}$, which is $\sim 150 \mathrm{meV}$ lower than III, where one reactant is in the inner-layer and one in the outer-layer. This latter in turn is $\sim 150 \mathrm{meV}$ lower than II, where both reactants are in the outer-layer. Based on the hydrophobic effects, reactants adsorbed on the surface are hence more prone to undergo addition reactions than reactants coming from the outer-layer. This result is very interesting and could explain why in the $\mathrm{CO}_{2}$ reduction to multi-carbon products, the formation of $\mathrm{C} 2$ intermediates from addition of two $\mathrm{CO}$ units usually occurs when both $\mathrm{CO}$ groups are adsorbed to the surface. ${ }^{17,36}$

This effect is more general and independent on the exact dimension: fixing the radii of $R_{1}$ and $R_{2}$ to a different value than $2.5 \AA$ leads to the same conclusions for the effect of the vertical distance from the surface, as shown in Supplementary Figure S2 for the $3.0 \AA$ case. The changes in $\Delta \mathrm{F}_{\text {reax }}$ become however more pronounced for increasing reactants radii.

\section{Effect of the size of the reactants}

In the following, the effect of the size of $R_{1}$ and $R_{2}$ at fixed distance of reactants and products from the surface is investigated by focusing on two different scenarios. In the first one, shown in Figure $4 \mathrm{~A}, \mathrm{R}_{1}$ is fixed in the inner layer while $\mathrm{R}_{2}$ and $\mathrm{P}$ in the outer-layer, and $\Delta \mathrm{F}_{\text {reax }}$ is evaluated as a function of the radii of $\mathrm{R}_{1}$ and $\mathrm{R}_{2}$. The difference in the size-effect of the two reactants 

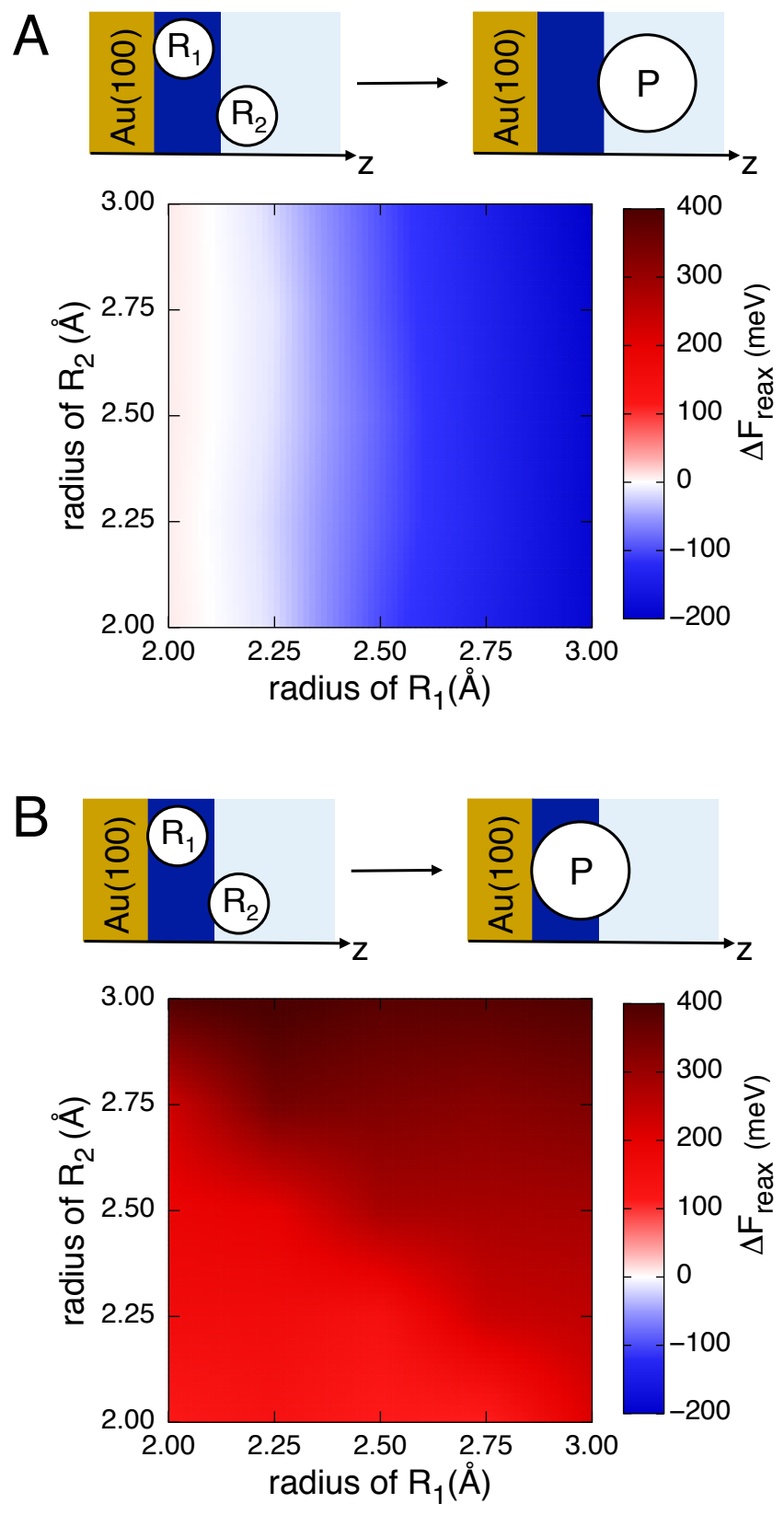

FIG. 4. A) $\Delta \mathrm{F}_{\text {reax }}$ (from dark blue to dark red) as a function of the radius of $R_{1}, x$-axis, and $R_{2}, y$-axis, for a fixed vertical distance of reactants and product: $\mathrm{z}_{R 1}-\mathrm{z}^{*}=-1.0 \AA, \mathrm{z}_{R 2}-\mathrm{z}^{*}$ and $\mathrm{z}_{P}-\mathrm{z}^{*}=0 \AA$. B) Same as A, but now $\mathrm{z}_{R 1}-\mathrm{z}^{*}$ and $\mathrm{z}_{P}-\mathrm{z}^{*}=$ $-1 \AA$ and $\mathrm{z}_{R 2}-\mathrm{z}^{*}=0 \AA$.

is striking. While the free energy strongly varies, from positive (red) to negative (blue) values as a function of the radius of $R_{1}$, it is almost insensitive to the size of $R_{2}$. This is due to the fact that $R_{2}$ is located in the outer-layer, i.e. same position as the product. Thus, any increase/decrease of its size (which also implies an increase/decrease of the size of $\mathrm{P}$ ) modifies the free energy cost to solvate the reactant and the product in a similar way, and the free energy of the model reaction is not significantly altered. On the contrary, increasing the radius of $R_{1}$, which occupies the inner-layer where the free energy cost for hydrophobic hydration is much larger, results in a greater destabilization of the hydration state of the reactants, thus favouring their addition reaction.

In the second scenario the position of $\mathrm{P}$ is in the inner-layer. As shown in Figure 4B, the effect of the size becomes now less prominent and hydrophobic hydration disfavours the addition reaction for any combination of $R_{1}$ and $R_{2}$ radii. Thus, the effect of the size of the reactants cannot be evaluated independently from their position, and it can provide a decisive contribution to $\Delta \mathrm{F}_{\text {reax }}$ in cases where the vertical distance from the surface is different between the product and at least one reactant.

\section{Application to "real" chemical reactions}

We now investigate how the hydrophobic hydration contributions rationalized in the previous sections affect the free energy of "real" electrochemical reactions occurring at the $\mathrm{Au} /$ water interface. In particular, we report in Figure 5 two examples; the first one (panel A) is the reduction of $\mathrm{N}_{2}$ to $\mathrm{NH}_{3}$, following the mechanism proposed from high-level DFT calculations ${ }^{31}$ and surface enhanced infrared absorption spectroscopy (SEIRAS). ${ }^{37}$ The second one (panel B) is a theoretically determined reaction pathway for the reduction of $\mathrm{CO}$ to ethylene. ${ }^{17}$

In both examples and for each reaction step, the free energy difference between reactants and products is given by the changes in the free energy of the reactive species, their interaction with the surface and their hydration. The first two terms were quantified in previous DFT calculations. ${ }^{17,31}$ However, due to the lack of explicit water, such calculations were not accounting for hydrophobic hydration free energies $\left(\Delta \mathrm{F}_{\text {cavity }}\right)$ at the interface. By using our adapted LCW theory, we can estimate $\Delta \mathrm{F}_{\text {cavity }}$ given the position and the size (see Methods for size estimation) of the cavities formed by the reactive species. In the figure, the molecules adsorbed to the surface, which form cavities in the inner-layer (at $\mathrm{z}^{-z^{*}}=-1.0 \AA$ ), are marked with $*$. The $\Delta \mathrm{F}_{\text {cavity }}$ values for the most relevant steps are highlighted with colored boxes.

In Figure 5A we show the $\mathrm{N}_{2}$ reduction mechanism. The first step (red box) involves the reductive adsorption of a $\mathrm{N}_{2}$ molecule from the bulk to the $\mathrm{Au}$ surface, with formation of the ${ }^{*} \mathrm{NNH}$ intermediate. This is predicted to be the rate determining step by DFT calculations ${ }^{31}$ and proposed to be at the origin of the low efficiency of the $\mathrm{N}_{2}$ reduction on different $\mathrm{Au}$ surfaces. According to what we have shown in Figure 2 , hydrophobic hydration contributes unfavourably to 

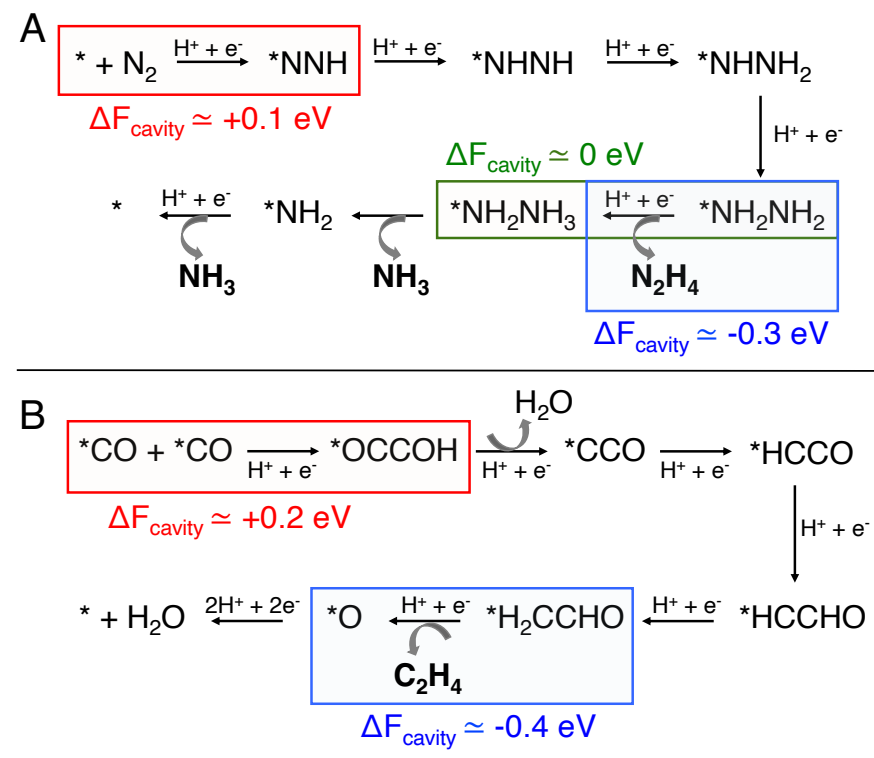

FIG. 5. A) Reaction pathway for the reduction of $\mathrm{N}_{2}$ to $\mathrm{NH}_{3}$ at the $\mathrm{Au} /$ water interface, as proposed in Refs. 31,37. The reaction step leading to the formation of hydrazine $\left(\mathrm{N}_{2} \mathrm{H}_{4}\right)$ byproduct is also reported. B) Reaction pathway for the reduction of $\mathrm{CO}$ to ethylene $\left(\mathrm{C}_{2} \mathrm{H}_{4}\right)$ at the $\mathrm{Au} /$ water interface, as proposed in Ref. 17. In both cases, molecules adsorbed on the surface (i.e. in the inner-layer, at $\mathrm{z}-\mathrm{z}^{*}=-1.0 \AA$ ) are marked with $*$, while the colored boxes identify the relevant reactions steps discussed in the text. The $\Delta \mathrm{F}_{\text {cavity }}$ values are the hydrophobic contributions to the associated reaction free energies, in $\mathrm{eV}$.

this step, since a free energy cost of $\sim 0.1 \mathrm{eV}$ is needed to bring the cavity formed by $\mathrm{N}_{2}$ from the bulk to the inner-layer. From the ${ }^{*} \mathrm{NNH}$ intermediate, the reaction continues exothermically until $* \mathrm{NH}_{2} \mathrm{NH}_{2}$ is formed. At this stage, which plays a crucial role for selectivity, the reaction can either proceed toward the formation of ${ }^{*} \mathrm{NH}_{2} \mathrm{NH}_{3}$ (green box) and then to the main product $\mathrm{NH}_{3}$, or follow an alternative path with the desorption of hydrazine $\left(\mathrm{NH}_{2} \mathrm{NH}_{2}\right)$ as byproduct (blue box). This latter step is predicted by DFT to be endothermic, with a free energy cost in the $0.2-0.7 \mathrm{eV}$ range (depending on the $\mathrm{Au}$ surface), while the reaction toward ${ }^{*} \mathrm{NH}_{2} \mathrm{NH}_{3}$ is exothermic. However, the formation of hydrazine during $\mathrm{N}_{2}$ reduction has been observed experimentally by SEIRAS. ${ }^{37}$ The hydrophobic hydration contribution offers a rationalization for this experimental result. For the reaction from ${ }^{*} \mathrm{NH}_{2} \mathrm{NH}_{2}$ to ${ }^{*} \mathrm{NH}_{2} \mathrm{NH}_{3}$, the size and position of reactant and product does not change, and the influence of the discussed hydrophobic hydration term is negligible. In contrast, hydrophobic hydration favours the desorption of hydrazine byproduct by $\sim-0.3 \mathrm{eV}$, and thus results in a more favorable free energy for the formation of hydrazine than previously anticipated.

In the second example (Figure 5B), we consider the addition reaction of two $\mathrm{CO}^{*}$ to form a $\mathrm{C}_{2}$ intermediate, ${ }^{*} \mathrm{OCCOH}$. In Ref. 17 , this is proposed to be the rate determining step for the reduction of $\mathrm{CO}$ to $\mathrm{C} 2$ products (ethylene and ethanol) occurring on strongly binding surfaces like $\mathrm{Pt}$, while the associated free energy cost is only $\sim 0.1 \mathrm{eV}$ for the weakly binding $\mathrm{Au}(100)$ surface. However, this free energy value substantially increases by $\sim 0.2 \mathrm{eV}$ once the hydrophobic hydration contribution is taken into consideration, see Figure 5B. Indeed, from the point of view of hydrophobic hydration, this reaction step corresponds to the unfavourable case of the addition of two cavities of $2.5 \AA$ radius that form a larger cavity of $3.25 \AA$ radius, in the inner-layer (see $[-1,-1]$ point in the 2D-map of Figure $3 \mathrm{~A})$. With the progress of the reaction, a second key step is reached once the ${ }^{*} \mathrm{H}_{2} \mathrm{CCHO}$ intermediate is formed. From this, an ethylene molecule desorbed from the surface is produced as a result of the elimination reaction step, as highlighted by the blue box. This step was predicted to be the rate determining step on $\mathrm{Au}(100)$, with a high free energy cost of $1.8 \mathrm{eV}$, as well as the step determining the selectivity between ethylene and ethanol products. ${ }^{17}$ For the release of $\mathrm{C}_{2} \mathrm{H}_{4}$ molecule from the inner-layer into the bulk, the $\Delta \mathrm{F}_{\text {cavity }}$ value estimated with the adapted LCW theory yields a favourable contribution to the reaction free energy, which is decreased by $\sim-0.4 \mathrm{eV}$ by the hydrophobic term.

The first step in $\mathrm{CO}_{2}$ reduction processes is usually the adsorption of the reactive molecule(s) from the bulk to the surface, which is omitted from the mechanism of Figure 5B despite it can become the rate determining step at $\mathrm{Au}$ surfaces at intermediate overpotentials. ${ }^{38}$ Hydrophobic hydration actively contributes to its free energy cost, as $\Delta \mathrm{F}_{\text {cavity }} \sim 0.2 \mathrm{eV}$ for the adsorption of a $\mathrm{CO}_{2}$ molecule (which forms in water a cavity of approximately $2.8 \AA$ radius $^{33}$ ) from the bulk to the inner-layer. Overall, this second example demonstrates how, by disfavouring both the adsorption of the reactive species to the $\mathrm{Au}$ surface and their addition, hydrophobic hydration contributions are detrimental to the $\mathrm{CO}_{2}$ reduction to multicarbon products. This provides an explanation for the low efficiency of the reaction on $\mathrm{Au}$ surfaces, where $\mathrm{CO}$ is the preferred product. ${ }^{39,40}$ However, once the $\mathrm{C} 2$ intermediate is formed, hydrophobic hydration contributes favourably, mitigating the high free energy cost for desorbing the ethylene product from the surface.

\section{CONCLUSIONS}

We have evidenced the importance of hydrophobic hydration contributions to chemical reactions occurring at the $\mathrm{Au}(100) /$ water interface that involve addition/elimination or adsorption/desorption steps. In particular, by applying our adaptation of the Lum-ChandlerWeeks theory ${ }^{22}$ to model chemical reactions between 
ideal spherical hydrophobes, we have found that both the size of the reactive species and their vertical distance from the surface modulate reaction free energies. We have mapped the conditions for which interfacial reactions can be either favoured or disfavoured by hydrophobic hydration. By considering two reaction pathways related to $\mathrm{CO}_{2}$ and $\mathrm{N}_{2}$ electrochemical reduction processes at the $\mathrm{Au} /$ water interface, we have shown that hydrophobic hydration free energies provide driving forces that actively contribute to reactions rate and selectivity. Such driving forces can be tuned with subtle modifications of the size of the reactive species and of the way they are adsorbed on the surface. These results open the way towards a quantitative understanding of how hydration free energies and hydrophobic effects can be exploited to modulate the energetics and mechanisms of chemical processes occurring at metal/water interfaces.

\section{SUPPLEMENTARY MATERIAL}

See the supplementary material for addition plots of the free energy of the model addition reaction discussed in the main text.

\section{ACKNOWLEDGMENTS}

We thank M. Salanne for fruitful discussions. M. H. and S. P. acknowledge financial support by ERC Advanced Grant 695437 THz-Calorimetry. This study is funded by the Deutsche Forschungsgemeinschaft (DFG,German Research Foundation) under Germany's Excellence Strategy-EXC2033-390677874-RESOLV. We thank the Mercator Research Center Ruhr (MERCUR) for funding.

${ }^{1}$ S. Nitopi, E. Bertheussen, S. B. Scott, X. Liu, A. K. Engstfeld, S. Horch, B. Seger, I. E. L. Stephens, K. Chan, C. Hahn, J. K. Nørskov, T. F. Jaramillo, and I. Chorkendorff, "Progress and perspectives of electrochemical co2 reduction on copper in aqueous electrolyte," Chem. Rev. 119, 7610-7672 (2019).

${ }^{2}$ J. D. Blakemore, R. H. Crabtree, and G. W. Brudvig, "Molecular catalysts for water oxidation," Chem. Rev. 115, 12974-13005 (2015).

${ }^{3}$ G. Qing, R. Ghazfar, S. T. Jackowski, F. Habibzadeh, M. M. Ashtiani, C.-P. Chen, M. R. Smith, and T. W. Hamann, "Recent advances and challenges of electrocatalytic $\mathrm{n} 2$ reduction to ammonia," Chem. Rev. 120, 5437-5516 (2020).

${ }^{4} \mathrm{~F}$. Meemken and A. Baiker, "Recent progress in heterogeneous asymmetric hydrogenation of $\mathrm{c}=\mathrm{O}$ and $\mathrm{c}=\mathrm{c}$ bonds on supported noble metal catalysts," Chem. Rev. 117, 11522-11569 (2017).

${ }^{5} \mathrm{~L}$. Liu and A. Corma, "Metal catalysts for heterogeneous catalysis: From single atoms to nanoclusters and nanoparticles," Chem. Rev. 118, 4981-5079 (2018).

${ }^{6} \mathrm{Q}$. Gu, P. Sautet, and C. Michel, "Unraveling the role of base and catalyst polarization in alcohol oxidation on au and pt in water," ACS Catal. 8, 11716-11721 (2018).

${ }^{7}$ A. A. Peterson and J. K. Nørskov, "Activity descriptors for co2 electroreduction to methane on transition-metal catalysts," J. Phys. Chem. Letters 3, 251-258 (2012).

${ }^{8}$ F. Abild-Pedersen, J. Greeley, F. Studt, J. Rossmeisl, T. R. Munter, P. G. Moses, E. Skúlason, T. Bligaard, and J. K.
Nørskov, "Scaling properties of adsorption energies for hydrogencontaining molecules on transition-metal surfaces," Phys. Rev. Lett. 99, 016105 (2007)

${ }^{9}$ F. Calle-Vallejo, J. I. Martínez, J. M. García-Lastra, J. Rossmeisl, and M. T. M. Koper, "Physical and chemical nature of the scaling relations between adsorption energies of atoms on metal surfaces," Phys. Rev. Lett. 108, 116103 (2012).

${ }^{10}$ F. Calle-Vallejo, D. Loffreda, M. T. M. Koper, and P. Sautet, "Introducing structural sensitivity into adsorption-energy scaling relations by means of coordination numbers," Nature Chem. 7, 403-410 (2012)

${ }^{11} \mathrm{P}$. R. Davies, "On the role of water in heterogeneous catalysis: A tribute to professor m. wyn roberts," Top. Catal. 59, 671-677 (2016).

${ }^{12}$ C.-R. Chang, Z.-Q. Huang, and J. Li, "The promotional role of water in heterogeneous catalysis: mechanism insights from computational modeling," WIREs Comput. Mol. Sci. 6, 679-693 (2016).

${ }^{13} \mathrm{~T}$. Andrushkevich and E. Ovchinnikova, "The role of water in selective heterogeneous catalytic oxidation of hydrocarbons," Mol. Catal. 484, 110734 (2020).

${ }^{14}$ W. Luo, X. Nie, M. J. Janik, and A. Asthagiri, "Facet dependence of co2 reduction paths on cu electrodes," ACS Catal. 6, 219-229 (2016).

${ }^{15}$ G. Marcandalli, A. Goyal, and M. T. M. Koper, "Electrolyte effects on the faradaic efficiency of co2 reduction to co on a gold electrode," ACS Catal. 11, 4936-4945 (2021).

${ }^{16}$ A. J. Garza, A. T. Bell, and M. Head-Gordon, "Mechanism of co2 reduction at copper surfaces: Pathways to 22 products," ACS Catal. 8, 1490-1499 (2018).

${ }^{17}$ S. Hanselman, M. T. M. Koper, and F. Calle-Vallejo, "Computational comparison of late transition metal (100) surfaces for the electrocatalytic reduction of co to c2 species," ACS Energy Lett. 3, 1062-1067 (2018).

${ }^{18}$ T. K. Todorova, M. W. Schreiber, and M. Fontecave, "Mechanistic understanding of co2 reduction reaction (co2rr) toward multicarbon products by heterogeneous copper-based catalysts," ACS Catal. 10, 1754-1768 (2020).

${ }^{19}$ I. Ledezma-Yanez, E. P. Gallent, M. T. Koper, and F. CalleVallejo, "Structure-sensitive electroreduction of acetaldehyde to ethanol on copper and its mechanistic implications for co and co2 reduction," Catal. Today 262, 90-94 (2016).

${ }^{20}$ A. Bagger, W. Ju, A. S. Varela, P. Strasser, and J. Rossmeisl, "Electrochemical co2 reduction: A classification problem," ChemPhysChem 18, 3266-3273 (2017).

${ }^{21}$ D. T. Limmer, A. P. Willard, P. Madden, and D. Chandler, "Hydration of metal surfaces can be dynamically heterogeneous and hydrophobic," Proc. Natl. Acad. Sci. 110, 4200-4205 (2013).

${ }^{22}$ A. Serva, M. Salanne, M. Havenith, and S. Pezzotti, "Size dependence of hydrophobic hydration at electrified gold/water interfaces," Proc. Natl. Acad. Sci. 118, e2023867118 (2021).

${ }^{23}$ K. Lum, D. Chandler, and J. D. Weeks, "Hydrophobicity at small and large length scales," J. Phys. Chem. B 103, 4570-4577 (1999).

${ }^{24} \mathrm{D}$. Chandler, "Interfaces and the driving force of hydrophobic assembly." Nature 437, 640-647 (2005).

${ }^{25}$ T. Cheng, H. Xiao, and W. A. Goddard, "Full atomistic reaction mechanism with kinetics for co reduction on cu(100) from ab initio molecular dynamics free-energy calculations at $298 \mathrm{k}$," Proc. Natl. Acad. Sci. 114, 1795-1800 (2017).

${ }^{26} \mathrm{H}$. H. Kristoffersen and K. Chan, "Towards constant potential modeling of co-co coupling at liquid water-cu(100) interfaces," J. Catal. 396, 251-260 (2021).

${ }^{27}$ X. Liu, P. Schlexer, J. Xiao, Y. Ji, L. Wang, R. B. Sandberg, M. Tang, K. S. Brown, H. Peng, S. Ringe, C. Hahn, T. F. Jaramillo, J. K. Nørskov, and K. Chan, "ph effects on the electrochemical reduction of $\mathrm{co}(2)$ towards $\mathrm{c} 2$ products on stepped copper," Nat. Commun. 10, 32 (2019).

${ }^{28}$ A. Bagger, L. Arnarson, M. H. Hansen, E. Spohr, and J. Rossmeisl, "Electrochemical co reduction: A property of the electro- 
chemical interface," J. Am. Chem. Soc. 141, 1506-1514 (2019).

${ }^{29}$ K. Jiang, R. B. Sandberg, A. J. Akey, X. Liu, D. C. Bell, J. K. Nørskov, K. Chan, and H. Wang, "Metal ion cycling of cu foil for selective c-c coupling in electrochemical co2 reduction," Nat. Catal. 1, 111-119 (2018).

${ }^{30}$ F. Creazzo and S. Luber, "Explicit solvent effects on (110) ruthenium oxide surface wettability: structural, electronic and mechanical properties of rutile ruo2 by means of spin-polarized dftmd," Appl. Surf. Sci. , 10.1016/j.apsusc.2021.150993 (2021).

${ }^{31}$ D. Bao, Q. Zhang, F.-L. Meng, H.-X. Zhong, M.-M. Shi, Y. Zhang, J.-M. Yan, Q. Jiang, and X.-B. Zhang, "Electrochemical reduction of $\mathrm{n} 2$ under ambient conditions for artificial n2 fixation and renewable energy storage using n2/nh3 cycle," Adv. Mater. 29, 1604799 (2017).

${ }^{32}$ K. Sharma and N. P. Adhikari, "Temperature dependence of diffusion coefficient of nitrogen gas in water: A molecular dynamics study," Int. J. Mod. Phys. B 28, 1450084 (2014).

${ }^{33}$ D. Jiao and S. B. Rempe, "Co2 solvation free energy using quasichemical theory," J. Chem. Phys. 134, 224506 (2011).

${ }^{34}$ T. S. van Erp and E. J. Meijer, "Ab initio molecular dynamics study of aqueous solvation of ethanol and ethylene," J. Chem. Phys. 118, 8831-8840 (2003).

${ }^{35}$ D. T. Kallikragas, K. I. Choudhry, A. Y. Plugatyr, and I. M. Svishchev, "Diffusivity and hydration of hydrazine in liquid and supercritical water through molecular dynamics simulations and split-flow pulse injection experiments," J. Chem. Phys. 139, 134507 (2013).

${ }^{36}$ J. H. Montoya, C. Shi, K. Chan, and J. K. Nørskov, "Theoretical insights into a co dimerization mechanism in co2 electroreduction," J. Phys. Chem. Letters 6, 2032-2037 (2015).

${ }^{37}$ Y. Yao, S. Zhu, H. Wang, H. Li, and M. Shao, "A spectroscopic study on the nitrogen electrochemical reduction reaction on gold and platinum surfaces," J. Am. Chem. Soc. 140, 14961501 (2018).

${ }^{38}$ S. Ringe, C. G. Morales-Guio, L. D. Chen, M. Fields, T. F. Jaramillo, C. Hahn, and K. Chan, "Double layer charging driven carbon dioxide adsorption limits the rate of electrochemical carbon dioxide reduction on gold," Nat. Commun. 11, 33 (2020).

${ }^{39} \mathrm{~S}$. Zhao, R. Jin, and R. Jin, "Opportunities and challenges in co2 reduction by gold- and silver-based electrocatalysts: From bulk metals to nanoparticles and atomically precise nanoclusters," ACS Energy Lett. 3, 452-462 (2018).

${ }^{40}$ A. Goyal, G. Marcandalli, V. A. Mints, and M. T. M. Koper, "Competition between co2 reduction and hydrogen evolution on a gold electrode under well-defined mass transport conditions," J. Am. Chem. Soc. 142, 4154-4161 (2020). 